\title{
Effects of products' weight and size on consumers' preference: focused on cookies
}

\author{
Taebeum Ryu (tbryu75@me.com)', Seon Yeong Park (parksy725@gmail.com), \\ Dayoon Seo (seodda@nate.com) ${ }^{1}$
}

${ }^{1}$ Department of Industrial and Management Engineering, Hanbat National University, Daejeon, 34158

\begin{abstract}
This study analyzed the preference according to the weight and size of the product in order to understand how the size-weight illusion occurred in the affect dimension. For this purpose, 54 students in their twenties who have proved their size estimation ability participated in the preference evaluation experiment on the various weights and sizes of cookie products. The cookie products, for which heaviness is considered positive, used in this experiment were well known to be sold in three paper boxes. In this experiment, nine kinds of cookie boxes were prepared by combining three sizes and three weight levels of this product. The participants evaluated the perceived heaviness of the cookie box by the modulus method and evaluated the preference of the weight with the 11-points semantic differential scale. As a result of the study, among the boxes with the same weight ones of small size is significantly heavier, and among the boxes with the same size ones of heavy weight is significantly heavier. This result mean that the size-weight illusion of the object heaviness was found to be the same in this study. Likewise, preference were similar, but this study found that it did not increase more than certain weight. That is, there was a limit to the preferred weight for one size cookie box.
\end{abstract}

Keywords: ESK-JES Joint Session, Product size and weight, Size-weight illusion, Cookie box, Preference, Heaviness

\section{Introduction}

In the sensory dimension, the weight of an object is known to be influenced by the weight of the object as well as the size, material, and color. According to Jones (1986), in the case of objects of the same weight but different sizes, we perceive heavier objects as heavier. This is explained by the fact that our brain commands a small amount of muscle force that is smaller than the required force for a small object, but requires more force than expected. On the contrary, in the case of a large object, the brain forces larger muscle force than the necessary force, but the force is less than expected and the object feels less heavy. The same principle applies to objects of the same weight but of different materials, which means that wooden objects are heavier than steel objects. Also, in the case of objects of the same weight but different colors, we feel darker objects lighter than light ones. In addition, Dijker (2008) reported that, in the study of the weight of a doll, the social factors such as the gender and age of the doll also affect the heaviness.

However, previous affective/Kansei studies, which focused on the weight of product, overlooked the size of product. Existing studies on the weight of products such as cell phones, clothing, and writing instruments do not suggest how the interaction between weight and size affects emotions. In addition, studies on the packaging/container weight of food products also focused on the weight of the container or the visual weight depending on the position of the product image on the packaging paper, but the size was not considered. Generally, the weight of a product is proportional to its size, so product design considering emotional satisfaction requires setting the size together with the weight. In addition, research that considers the weight and size of a product can show preference weight according to the size, that is, the preferred density of the product.

Therefore, this study grasps consumers' preference according to product weight and size and suggests preference weight according to product size. For this purpose, this study selected cookies products which are preferred weight and examined the preference of cookies with various weight and size. This study assumes that consumers' preference increases with the weight of the cookie product, but it does not increase if it is excessively heavy.

\section{Method}




\subsection{Participants}

Sixty-one college students (41 males and 20 females) voluntarily participated in this study. All of them did not have any physical or mental problems in assessing the weight of the object. Of these, 54 (38 males and 16 females) had passed the evaluation of the ability to estimate the magnitude of the line length. Lodge (1981, cited in Han et al. (1999)) reported that about 3-6\% of the population did not pass the size estimation ability test.

\subsection{Selection of cookie products}

In this study, we selected cookies which are expected to have high preference according to the weight of the products. Confectionery products related to health were excluded from the study, assuming that weight and preference were not correlated or negatively correlated. The experimental cookie products were sold in at least three sizes and packed in paper boxes that have a large weight range and are easy to handle. The small size box of this product had a volume of $535.44 \mathrm{~cm}^{3}(13.8,9.7,4 \mathrm{~cm})$, a medium size box of $1035.72 \mathrm{~cm}^{3}(18.3,13.7,4 \mathrm{~cm})$ and a large box of $2067 \mathrm{~cm}^{3}(26.5,19.5,4 \mathrm{~cm}$. And these boxes weighed 100, 198, and 385 grams from the small ones.

Therefore, in this experiment, nine kinds of boxes were prepared by combining three weights and sizes. The contents were removed so that one size box had three weights or weights were adjusted using weights.

\subsection{Procedure}

First, the magnitude estimation ability of participants, which is shown in Han et al. (1999), was confirmed by a preliminary experiment that estimates the number as a line length and the line length as a number. Whether or not the estimating ability was evaluated by evaluating whether the $95 \%$ confidence interval of the regression equation after the logarithmic conversion of the line length and the number includes 1 .

Participants who had passed the preliminary experiment were informed about the purpose of the experiment, the method, and the evaluation items, and evaluated the heaviness and preference of the cookie box. In this experiment, heaviness of the box was evaluated by modulus method based on weight value of reference box. This method can be used when the difference between the reference stimulus and the test stimulus is not large (Han et al., 1999). In this experiment, the reference stimulus was selected as a $198 \mathrm{~g}$ box which did not change the weight of medium size among the nine boxes. The heaviness of the $198 \mathrm{~g}$ box was set at 100 . The reference box was prepared separately from the evaluation boxes and was placed next to the evaluator to be used before the box evaluation. The preference for the weight of cookies was measured using semantic differential method.

\section{Results}

\subsection{Effect of weight and size}

The results of this study are as follows. First, the results of the analysis of variance of weight and size showed significant effects on weight, preference. The weight had a significant effect on the heaviness $(\mathrm{F}(2,106)=234.99, \mathrm{p}$ $<0.001)$ and preference $(\mathrm{F}(2,106)=45.46, \mathrm{p}<0.001)$. Also size had a significant effects on the heaviness $(\mathrm{F}(2,106)=$ $82.02, \mathrm{p}<0.001)$, preference $(\mathrm{F}(2,106)=33.68, \mathrm{p}<0.001)$. The interaction between weight and size was also significant.

\subsection{Analysis of heaviness difference according to weight and size}

As shown in Fig. 1, the heaviness of the small objects tended to increase more as the weight increased. The simple effect test was used to compare the heaviness differences between different sizes at different weight levels. LSD (Least Significant Difference) analysis showed that there was a difference in heaviness between all sizes at each weight level $(p<0.001)$. At the same weight level, the heaviness of small box was significantly higher than that of middle box and big box, and the heaviness of middle box was significantly higher than that of big box. That is, the smaller the size, the greater the heaviness was, and the tendency became worse as the weight increased. 


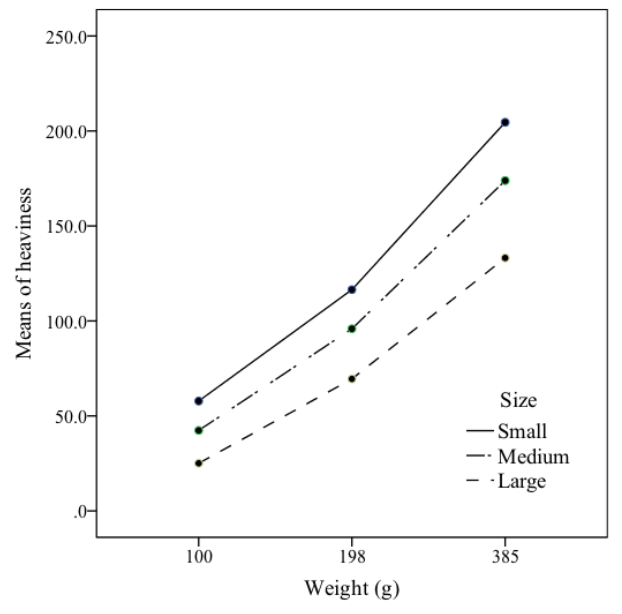

Figure 1. Interaction effects of size and weight of cookie box on heaviness

\subsection{Analysis of preference difference according to weight and size}

As shown in Figure 2, the weight and size interaction in preference increases as the weight increases in the large box, but not in the small box. The preference of $385 \mathrm{~g}$ was significantly higher than that of $198 \mathrm{~g}$ and $100 \mathrm{~g}$, and the preference of $198 \mathrm{~g}$ was significantly higher than that of $100 \mathrm{~g}$. It was confirmed that the preference increases as the size increases. The middle box also had the same results as the big box, but the difference between $198 \mathrm{~g}$ and $385 \mathrm{~g}$ was not as large as the box. On the other hand, the preference for a weight of $198 \mathrm{~g}$ in a small box was significantly higher than that of $100 \mathrm{~g}$ (difference between $198 \mathrm{~g}$ and $385 \mathrm{~g}$ in an intermediate box), but the preference of $385 \mathrm{~g}$ weight was not statistically different from that of $198 \mathrm{~g}$, and its level was similar. These results show that the preference does not increase as the weight increases in one cookie box size, and there is a preferred limit weight. As a result of comparing the preference according to the size of box at each weight, the preference of the box size at $100 \mathrm{~g}$ and $198 \mathrm{~g}$ was significantly increased as the box size was smaller, but the preferences of the three size boxes at $385 \mathrm{~g}$ were all the same.

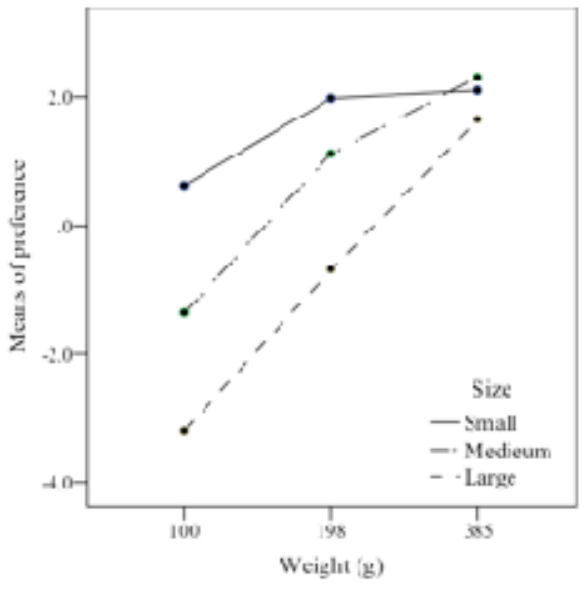

Figure 2. Interaction effects of size and weight of cookie box on preference

\subsection{Preference by box density}

In this study, the relationship between the box density and the average preference of the experimental participants is shown as a scatter plot (Figure 3) to show the limit weight of preference according to box size. At this time, density and $\log$ conversion were performed without preference. Preference linearly increased to $0.37 \mathrm{~g} / \mathrm{cm} 3$ (Log Conversion value-0.43) but not to the highest density $\left(0.72 \mathrm{~g} / \mathrm{cm}^{3}\right) \quad$ (Log Conversion-0.14). The regression equation of preference is preference $=5.14+6.06 \mathrm{log}$ (Density). Its coefficient of determination is 0.94 . This result implies that the weight of a certain cookie box size is determined by using the density $0.37 \mathrm{~g} / \mathrm{cm}^{3}$ proposed in this study, and the preference is high.

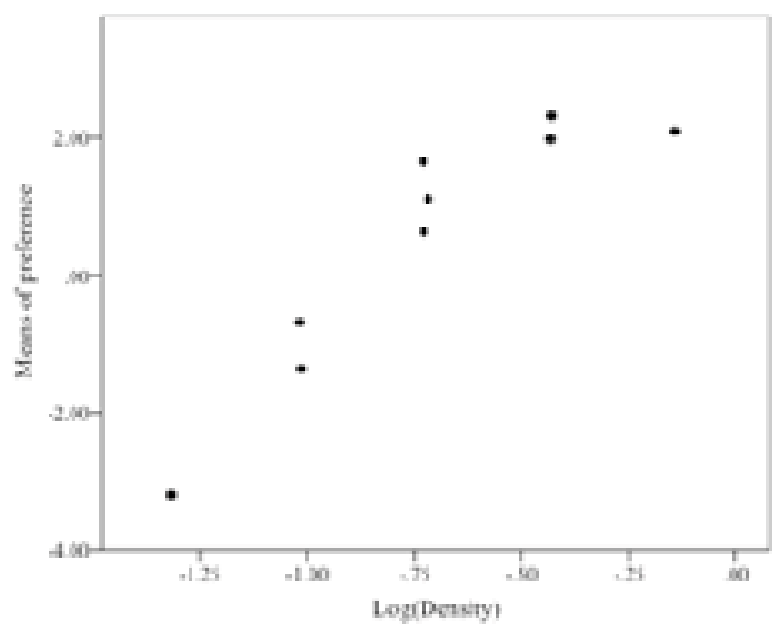

Figure 3. Scatter plot between preference and cookie box density 


\section{Conclusion}

This study analyzed the preference of the affective dimension according to the weight and size of the cookie box together with the weight of sensory dimension. This study experimentally found that there is a limit on the preference of the cookie box, which is positively correlated with the weight and positive correlation in the size of the cookie box. The present study analyzed the preference according to the density and suggested a limit density of 0.37 $\mathrm{g} / \mathrm{cm}^{3}$ in order to simplify the preference limit weights according to the box size. The limit density suggested in this study could be used to determine the weight of the cookie box.

\section{Acknowledgements}

This research was supported by the Basic Science Research Program through the National Research Foundation of Korea (NRF) funded by the Ministry of Education (NRF-2016R1D1A1B01009516).

\section{References}

Dijker A.J.M. Why Barbie feels heavier than Ken: The influence of size-based expectancies and social cues on the illusory perception of weight, Cognition, 106, 1109-1125, 2008.

Han, S. H., Song, M., \& Kwahk, J. A systematic method for analyzing magnitude estimation data, International Journal of Industrial Ergonomics, 23(5-6), 513-524, 1999.

Jones, L.A. Perception of force and weight: Therory and research, Psychological Bulletin, 100(1), 29-42, 1986. 